\title{
Educación corporativa: análisis del índice de contribuciones y desarrollo de competencias organizacionales
}

DOI: https://doi.org/10.21158/01208160.n87.2019.2443

Fecha de recepción: 21 de diciembre de 2018

Fecha de aprobación: 05 de diciembre de 2019
Lesley Carina do Lago Attadia Galli Universidade Estadual Paulista "Júlio de Mesquita Filho"

lesley.attadia@unesp.br

Glaucia Aparecida Prates

Universidade Estadual Paulista "Júlio de Mesquita Filho" g.prates@unesp.br

Caroline Aline Melenchon

Universidade Estadual Paulista "Júlio de Mesquita Filho" caamelenchon@gmail.com

Patrícia Andrea da Silva

Universidade Estadual Paulista "Júlio de Mesquita Filho" patricia.andrea@unesp.br

Rafael Altafin Galli Centro Universitário Estácio de Ribeirão Preto rafelaltafin@hotmail.com

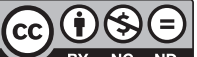

Cómo citar este artículo / To reference this article / Comment citer cet article / Para citar este artigo:

Attadia Galli, L. C.; Prates, G. A.; Melenchon, C. A.; Da Silva, P. A.; Altafin Galli, R. (2019). Educación corporativa: análisis del índice de contribuciones y desarrollo de competencias organizacionales. Revista Escuela de Administración de Negocios, (87), 43-57. DOI: https://doi.org/10.21158/01208160.n87.2019.2443

\section{Resumen}

Las organizaciones se encuentran involucradas en constantes cambios y buscan implementar estrategias que les permitan enfrentar los nuevos desafíos que generan, sobre todo, la globalización y la competitividad, centrados en el poder del conocimiento. El objetivo de este artículo es mapear las contribuciones de la educación corporativa en la formación y el desarrollo de las competencias de los colaboradores de una organización del sector bancario. Para la realización de este trabajo se optó por una muestra no probabilística y por conveniencia. El cuestionario se aplicó en trabajadores del Banco ALFA de las ciudades de Ribeirão Preto, Jaboticabal e Itu, de Brasil. Lo contestaron 200 funcionarios en total. De esta manera, fue posible concluir que los participantes concuerdan, parcialmente, en que el banco valora los funcionarios que buscan entrenamiento, por tanto, para

${ }^{1}$ Graduada en Administración - FEA- Universidade de Sao Paulo (USP) - Ribeirao Preto/SP - Brasil. Magíster en Ingeniería de producción - Universidade Federal de Sao Carlos (UFSCar) - Sao Carlos/SP - Brasil. Doctora en Administración - FEAUniversidade de Sao Paulo (USP) - Ribeirao Preto/SP - Brasil. ORCID: https://orcid.org/0000-0001-6641-9021

${ }^{2}$ Graduada en Ingeniería Forestal - Universidade Federal de Viçosa (UFV) - Brasil. Magíster en Ingeniería Forestal - Universidade Federal do Paraná (UFPR) - Brasil. Doctora en Ingeniería de Producción y Sistemas - Universidade Federal de Santa Catarina (UFSC) - Brasil. ORCID: https://orcid.org/0000-0002-8916-3441

${ }^{3}$ Graduada en Administración - Universidade Estadual Paulista "Júlio de Mesquista Filho" - Jaboticabal/SP - Brasil. ORCID: https:// orcid.org/0000-0003-1886-9102

${ }^{4}$ Graduada en Tecnología en Análisis y Desarrollo de Sistemas - FATEC Taquaritinga/SP - Brasil. ORCID: https://orcid.org/00000001-5775-5883

${ }^{5}$ Graduado en Derecho - UNAERP - Universidade de Ribeirao Preto/SP - Brasil. Magíster en Derecho - UNAERP - Universidade de Ribeirao Preto./SP - Brasil. ORCID: https://orcid.org/0000-0002-7512-4265 
ellos poseer capacitaciones no es un diferencial profesional frente a otro colaborador que no los posea. Sin embargo, el análisis de los resultados evidenció que es esencial un mayor incentivo a la participación en los programas o cursos financiados por el banco; pues queda clara la importancia y la necesidad de una adecuada implementación de procesos de educación corporativa, pues esta se presenta como un pilar fundamental para una gestión empresarial exitosa.

Palabras clave: educación corporativa; capacitación corporativa; capacitación laboral; gestión del conocimiento; competencias organizacionales; innovación organizacional.

\title{
Corporate Education: Analysis of the Contribution Index and Organizational Competency Development
}

\begin{abstract}
Organizations are involved in constant change, and seek to implement strategies that allow them to face the new challenges that are generated, above all, by globalization and competitiveness, centered on the power of knowledge. The aim of this article is to map the contributions of corporate education to the training and development of competencies of the employees working in an organization in the banking sector. For the development of this work, we chose a non-probability convenience sample. The questionnaire was applied to workers from Banco ALFA in the cities of Ribeirão Preto, Jaboticabal, and Itu, in Brazil. A total of 200 employees answered the questionnaire, thus, it was possible to conclude that the participants partly agree with the fact that the bank values the employees who seek for the opportunity of training; so for them, having training is not a professional differential against another employee who does not have it. However, the analysis of the results showed that it is essential to work on a greater incentive for employees to participate in the programs or courses that are financed by the bank; as the importance and the necessity of an adequate implementation of corporate education processes becomes clear, taking into account that it is presented as a fundamental pillar for a successful corporate management.
\end{abstract}

Keywords: corporate education; corporate training; job training; knowledge management; organizational competencies; organizational innovation. 


\section{Educação corporativa: análise do índice de contribuição e desenvolvimento de habilidades organizacionais}

\section{Resumo}

As organizações se encontram em constante mudanças e buscam implementar estratégias que lhes permitam enfrentar os novos desafios gerados, sobretudo, pela globalização e competitividade, focados no poder do conhecimento. $\mathrm{O}$ objetivo deste artigo é mapear as contribuições da educação corporativa no treinamento e desenvolvimento das habilidades dos funcionários de uma organização no setor bancário. Para a realização deste trabalho, foi escolhida uma amostra não probabilística e de conveniência. O questionário foi aplicado a trabalhadores do Banco ALFA nas cidades de Ribeirão Preto, Jaboticabal e Itu, no Brasil. Um total de 200 funcionários responderam. Dessa forma, foi possível concluir que os participantes concordam parcialmente que o banco valoriza os funcionários que buscam treinamento, portanto, para eles, ter treinamento não é um diferencial profissional em comparação com outro colaborador que não. No entanto, a análise dos resultados mostrou que é essencial um maior incentivo para participar de programas ou cursos financiados pelo banco porque é clara a importância e a necessidade de uma implementação adequada dos processos de educação corporativa, uma vez que este é apresentado como um pilar fundamental para o sucesso do gerenciamento de negócios.

Palavras-chave: educação corporativa; treinamento corporativo; formação profissional; Gestão do conhecimento; habilidades organizacionais; inovação organizacional.

\section{Formation en entreprise: analyse de l'indice de contribution et développement des compétences organisationnelles}

\section{Résumé}

Les organisations sont impliquées dans des changements constants et cherchent à mettre en œuvre des stratégies leur permettant de faire face aux nouveaux défis créés par la mondialisation et la compétitivité et centrés autour du savoir. L'objectif de cet article est de cartographier les contributions de la formation en entreprise à la formation et au développement des compétences des collaborateurs d'une organisation du secteur bancaire. Pour effectuer cette investigation nous avons choisi un échantillon non probabiliste de commodité. Le questionnaire a été soumis aux employés de la banque ALFA des villes de Ribeirão Preto, Jaboticabal et Itu, au Brésil. Au total deux cents collaborateurs y ont répondu s'accordant en partie sur le fait que la banque valorise les employés en quête de formation mais ne marque pas de différentiel professionnel par rapport à un autre collaborateur moins formé. L'investigation montre par ailleurs des résultats suggérant une plus grande incitation à participer aux programmes financés par la banque. L'importance et la nécessité d'une mise en œuvre adéquate des processus de formation en entreprise sont claires car présentées comme un pilier fondamental pour une gestion d'entreprise réussie.

Mots-clés: formation en entreprise; formation professionnelle; gestion des connaissances; compétences organisationnelles; innovation organisationnelle 


\section{Introducción}

Tas organizaciones están repensando la cuestión Lde la educación en lo que se refiere al ambiente corporativo. De esta manera, encuentran, en una de sus vertientes, a la universidad corporativa. De acuerdo con Esteves y Meiriño (2015), este modelo de educación, alineado con las nuevas tecnologías, comprende los diversos tipos de enseñanza, entre los que se encuentran los cursos técnicos, la educación básica, de posgrado y otros.

Las organizaciones modernas exigen nuevos valores, parámetros y prácticas administrativas (Chiavenato, 2009, p. 148). Muchos estudiosos consideran a la educación corporativa como la mejor manera de vincular los programas de aprendizaje a las metas y los resultados estratégicos de la empresa, con lo cual apuntan no solo a la sobrevivencia de la empresa, sino también a su expansión futura.

Para Garbanzos-Vargas (2015), la capacidad organizacional de aprendizaje, por sí misma, es una innovación que orienta el desarrollo organizacional. Además, esta influye de forma positiva en el desarrollo de las innovaciones y en una gestión competitiva organizacional.

Se observa que el papel de las organizaciones en el aprendizaje de sus funcionarios se encuentra en un proceso de expansión constante. La educación corporativa focaliza el desarrollo del cuadro de personal con vistas a la obtención de los resultados en los negocios. Se trata de un modelo de entrenamiento diferenciado, estructurado tanto para transmitir conocimientos específicos sobre asuntos en los cuales los funcionarios presentan algunas deficiencias como en el propósito de preparar a estos funcionarios para los desafíos venideros (Amorim, Cruz, Sarsur y Fischer, 2015).

Las organizaciones hacen frente a las innovaciones tecnológicas y organizacionales relacionadas con las nuevas formas de organización del trabajo que se encuentran involucradas en constantes cambios y buscan implementar políticas y estrategias que las vuelvan más competitivas; esto las lleva también a anticiparse y prepararse para enfrentar los nuevos desafíos generados, sobre todo, por los desdoblamientos de la globalización y de sus bases de competitividad, centrados en el poder del conocimiento.

Según Vega y Salaiza (2016), los nuevos enfoques de organización buscan crear un ambiente propicio que permita agilizar y dar mayor rapidez a las respuestas con el fin de, finalmente, generar conocimiento de manera grupal.

La educación corporativa intenta cubrir los espacios que ha dejado la academia y concentrar sus contenidos en desafíos específicos, así como en la complejidad del ambiente empresarial (Febraban, 28 de agosto de 2017).

El objetivo de este artículo es mapear las contribuciones de la educación corporativa en la formación y el desarrollo de las competencias de los colaboradores de una organización del sector bancario. 


\section{Universidades corporativas}

A llen (2002) define a la universidad corporativa como una entidad educacional que es a la vez una herramienta estratégica diseñada con el propósito de ayudar a la compañía matriz a alcanzar su misión mediante la realización de actividades que cultivan el aprendizaje, el conocimiento y la sabiduría individual y organizacional.

De acuerdo con Montaña, Labrador y Jiménez (2016), la universidad corporativa en cuanto departamento estratégico en la compañía tiene como objetivo el logro de la intención de la empresa por medio de actividades que generan conocimiento a través de la formación en el aprendizaje individual y organizacional.

Meinster (1999) define universidad corporativa como «un paraguas estratégico para el desarrollo y la educación de los funcionarios, clientes y proveedores, buscando optimizar las estrategias organizacionales, además de ser un laboratorio de aprendizaje para las organizaciones de un espacio permanente».

Grandes compañías han creado su universidad corporativa a lo largo de los últimos años. Sin embargo, la primera universidad corporativa de la que se tiene conocimiento la creó en 1955 General Electric (Tavares-Filho y Bernardes, 2005). Según Montaña et al. (2016), Walt Disney creó su universidad en 1955, Toyota otra en 1988 y Ecopetrol la suya en el 2007.

En Brasil, las primeras universidades corporativas creadas fueron las de Brahma - una empresa brasileña del sector de cervezas - y Arcor - una empresa argentina del sector de chocolates-, en la década de los noventa del siglo XX, bajo la presión de la competitividad del mercado y con miras al crecimiento de sus negocios institucionales (Moraes, 2016).

La principal función de la universidad corporativa es conseguir que sus programas formativos apoyen los intereses estratégicos de la organización y, además, alinear el desarrollo de los trabajadores con los objetivos del negocio (Iglesias, 2017).

La universidad corporativa es el modelo de uso más común de la aplicación del concepto de educación corporativa. Actúa como una unidad independiente de negocios e incluye las metas de educación, entrenamiento y desarrollo de los integrantes de la cadena productiva y de relacionamiento de la organización con sus resultados estratégicos, previendo el retorno financiero de las inversiones; es decir, sostenerse y generar lucros como las demás unidades de negocios de la organización (Martino y Scarcella-Prandstraller, 2018).

Chase (2016) se enfocaron en la justificación teórica de tipos y formas de educación corporativa de especialistas en emprendimientos modernos y en el desarrollo de su clasificación en escala local, finalidad temporal cuyas peculiaridades son divulgadas en los tipos y las formas de entrenamiento corporativo presentados. Su selección de tipos y formas en el proceso de entrenamiento corporativo es resultado de condiciones socioeconómicas y organizacionales-pedagógicas de las empresas dirigidas a implementar el entrenamiento de sus funcionarios.

Dahyana y Susanty (2018) midieron el efecto del sistema de incentivo en la motivación del funcionario, el efecto de motivación para el desempeño del funcionario y el efecto del sistema de incentivo directamente enfocado en el desempeño del funcionario en una universidad corporativa. El proceso de datos usa el SPSS 20 o Statistical Package for the Social Sciences, en español Paquete Estadístico para las Ciencias Sociales, una herramienta de investigación desarrollada por la empresa IBM, que analiza mediante regresión para múltiplos. La muestra de este estudio fue de 200 colaboradores. Los datos se colectaron usando un cuestionario que consiste en 55 ítems, lo que incluye declaraciones de sistema de incentivo, motivación y desempeño individual. 
El resultado de este estudio mostró que el sistema de incentivo tiene impactos positivos y significativos para los funcionarios motivados. Pues la motivación de los colaboradores impacta de forma positiva y significativa su desempeño (Mazariegos, 2015).

Por otra parte, el sistema de incentivo no presentó impacto significativo directo para el desempeño de los funcionarios en una universidad corporativa en Indonesia. Por tanto, una universidad corporativa debe considerar el sistema de incentivo como un factor motivador a fin de influenciar el desempeño de los trabajadores.

La gestión de la universidad corporativa debe crear un sistema de incentivo atrayente y eso motivará a los funcionarios. El sistema de incentivos no se concentra tan solo en el incentivo financiero, sino que también considera los beneficios no financieros. De acuerdo con Chiavenato, «cada persona aprende con una amplia variedad de medios, según las necesidades del momento y los contenidos que asimilará» (2009, p. 156).

Así, la universidad corporativa integra varios sistemas de gestión de personas y de educación corporativa $y$, por tanto, es posible decir que constituye un segmento de actuación del área de gestión de personas (Brandão, 2006).

Cappiello y Pedrini (2017) ilustran el fenómeno de las universidades corporativas a partir de la perspectiva de la evaluación de su desempeño. Las universidades corporativas tienen una naturaleza híbrida que se puede referir como una unidad de negocios y una institución de enseñanza superior. Los resultados mostraron que una estructura apropiada para la evaluación del desempeño de las universidades corporativas precisa distinguir entre los diferentes tipos de universidades corporativas que están realmente en vigor, de acuerdo con su permisión y el grado de apertura hacia el ambiente externo.

Chaise (2016), por su parte, estudió la necesidad de crear una solución de educación orientada a capacitar, en un periodo de siete meses, a empleados de la Empresa Brasileña de Correos y Telégrafos que trabajan en atención al público, quienes deberían certificarse en operaciones de créditos. El desafío de la universidad corporativa era impulsar la motivación y propiciar el aprendizaje de todos estos empleados. El foco de este proyecto fue registrar la forma en que se desarrolló la solución de educación para capacitar los empleados de las 28 regiones de Brasil.

Ahora bien, este proyecto buscó también comprender por qué, en ciertas regiones, las prácticas no fueron aparentemente exitosas y cuáles son los problemas que ocasionaron tal situación, caracterizada por el factor de los resultados obtenidos que no demostraron ser satisfactorios. Este proceso investigativo se realizó por medio de un cuestionario on line, el cual contestaron los apoyadores regionales mediante el método deductivo e inductivo. Se obtuvieron buenos resultados en cuanto a la modalidad de capacitación, la actuación de la universidad corporativa y la gestión estratégica. También se evidenciaron fragilidades con respecto a la actuación del gestor inmediato, la calidad en la red de datos y la poca desenvoltura por parte del alumno con relación al dominio de tecnologías digitales.

En consideración a las fragilidades de este sistema, consecuentemente, «un sistema de evaluación sólido puede hacer la diferencia en la percepción del valor y credibilidad de una universidad corporativa al mostrar que ella contribuye para los resultados» (Guerci et al., 2011, p. 292).

Martino y Scarcella-Prandstraller (2018) exploraron las tendencias emergentes y los abordajes estratégicos promovidos por bancos italianos en el campo de la responsabilidad cultural corporativa y la comunicación. Con base en un abordaje de estudio de casos múltiples, la investigación enfocó tanto las políticas como las actividades realizadas en la última década por algunos de los actores más innovadores del área de gestión y comunicación corporativa, con lo cual promovió una lógica innovadora de colaboración social y compromiso de las partes interesadas en torno a los eventos y las plataformas digitales.

En relación con el actual ambiente de negocios y sus reflejos sobre las organizaciones, se destaca como primer aspecto la emergencia de una sociedad basada 
en el conocimiento, lo cual se manifiesta en la forma de un acelerado desarrollo tecnológico en el que la tecnología de la información adquiere un papel importante. Las transformaciones, según Vergara (2000), se pueden agrupar en torno a tres áreas: tecnológica, humana y organizacional.

Las transformaciones de orden tecnológico se manifiestan en las organizaciones a través de nuevas formas de trabajo, en el flujo y el tratamiento de las informaciones, en la individualización de productos y servicios, y en el énfasis en servicios adicionales.
Los cambios en la dimensión humana se manifiestan en el compartimento de responsabilidades en busca de mayor participación y autonomía, confianza y ética, que son pilares inamovibles.

La otra transformación comprende la adecuación organizacional, la cual se verifica a través de la reducción de los niveles jerárquicos, por medio de la horizontalización de las estructuras, el surgimiento de las estructuras en red o cadena escalar y de las organizaciones virtuales.

\section{Metodología}

$\mathrm{P}$ ara la consecución del objetivo se realizó una investigación de naturaleza exploratorio-descriptiva y un abordaje cuantitativo. Como método de investigación se utilizó el levantamiento de datos.

El método de recolección de datos elegido fue el cuestionario estructurado, compuesto de tres partes: perfil del bancario, caracterización de las actividades de educación corporativa y evaluación de la contribución de las actividades de educación corporativa. La tabla 1 presenta las principales variables de investigación de cada uno de los aspectos evaluados.

Tabla 1. Variables de investigación

\begin{tabular}{|c|c|}
\hline Aspectos evaluados & Variables de investigación \\
\hline $\begin{array}{l}\text { Caracterización de } \\
\text { las actividades de } \\
\text { educación corporativa }\end{array}$ & $\begin{array}{l}\text { - La manera como son realizadas la mayoría de las actividades de actualización/capacitación. } \\
\text { - El motivo de la participación en los cursos. } \\
\text { - La participación en los cursos financiados parcialmente o integralmente por el Banco } \\
\text { ALFA. } \\
\text { - La cantidad, el tiempo medio de duración, la regularidad de acceso a los cursos vía intranet } \\
\text { y a los cursos presenciales. }\end{array}$ \\
\hline $\begin{array}{l}\text { Evaluación de la } \\
\text { contribución de } \\
\text { las actividades de } \\
\text { educación corporativa }\end{array}$ & $\begin{array}{l}\text { - La intensidad de los cambios de orden tecnológico, humano y organizacional en el día a } \\
\text { día. } \\
\text { - La conducción de la realización de los cursos. } \\
\text { - La opinión general sobre la forma en la que los cursos se conducen. } \\
\text { - La intención del Banco ALFA frente a la disponibilidad de los cursos. } \\
\text { - Las complicaciones para su realización. } \\
\text { - La contribución al crecimiento personal, profesional y organizacional. } \\
\text { - Las ventajas y desventajas, las competencias y las habilidades desarrolladas con la } \\
\text { realización de los cursos vía intranet y presencialmente. } \\
\text { - Oportunidad de entrenamiento y capacitación - enfoque a corto plazo-. } \\
\text { - Desarrollo profesional - enfoque a largo plazo-. } \\
\text { - Intercambio de conocimientos con colegas de trabajo y con el superior inmediato, y de } \\
\text { experiencias entre los equipos de trabajo. } \\
\text { - Cooperación y auxilio entre los colegas de trabajo y el superior inmediato en la ejecución } \\
\text { de las actividades. } \\
\text { - Evaluaciones, feedbacks e informaciones sobre desempeño. }\end{array}$ \\
\hline
\end{tabular}

Fuente. Elaboración propia. 
En relación con el pretest del cuestionario, hubo cambios en apenas una pregunta que se transformó en semiabierta para una mejor comprensión. Es relevante informar que los cuestionarios del pretest se incorporaron a la muestra.

La aplicación del cuestionario se finalizó en la segunda quincena de octubre del 2016, en su mayoría, en el horario de trabajo de los sectores bancarios, que fluctuaba entre las 10:00 y las 15:00 en las ciudades de Ribeirão Preto, Jaboticabal e Itu.
Para la realización de este trabajo se optó por una muestra no probabilística y por conveniencia. El cuestionario se aplicó junto con los colaboradores del Banco ALFA, el cual se encontraba compuesto por 200 funcionarios de la organización financiera, de los cuales 184 funcionarios y 16 practicantes pertenecían a las agencias del Banco ALFA ubicadas en las ciudades paulistas de Ribeirão Preto, Jaboticabal e Itu.

\section{Resultados y discusiones}

$E^{1}$ Banco ALFA realiza inversiones en el desarrollo de líderes, en la capacitación de gestores y en entrenamientos dirigidos a toda la red de agencias. Mantiene programas corporativos que comprenden apoyo a los procesos de educación formal tales como graduación, posgraduación, MBA y cursos de idiomas, así como en temas transversales, como, por ejemplo, colaboración, sustentabilidad, diversidad, compliance y riesgo.

Todas las ocho agencias bancarias estudiadas poseen acceso al mismo programa de capacitación, entrenamientos y desarrollo, sin excepción. Para esto, el Banco ALFA desarrolla prácticas que apuntan a proporcionar desarrollo personal y profesional a sus funcionarios. Se observan también los siguientes programas de capacitación: Capacitación de Gestores, Programa Carreras, Futuros Directivos, y ATEP ALFA Executive Training Programme o Programa de Entrenamiento Ejecutivo ALFA-.

\subsection{Capacitación de funcionarios}

Frente a la vasta opción de programas de capacitación es posible percibir que se realizan esfuerzos de la organización bancaria con relación al desarrollo de sus funcionarios. Se invierten anualmente más de BRL 86 millones de reales brasileños, esto es, cerca de USD
22 millones de dólares. Son 1,74 millones de horas de entrenamiento, esto es, cerca de 33,5 por persona al año, más de 326000 participaciones, es decir, 6,1 por persona al año, más de 300 cursos en línea y 350 cursos presenciales.

La capacitación de estos funcionarios ocurre a través de cursos presenciales y a distancia. Todo funcionario contratado pasa por un entrenamiento presencial de capacitación de una semana para la función que realizará, en San Pablo, sede de la organización. Allí, además de interactuar con la cultura organizacional, asiste a charlas realizadas por funcionarios capacitados específicamente para el entrenamiento.

La gran revolución generada por esta forma de comunicación es, sin duda, la facilidad de acceso a la información. En una intranet la localización de la información se torna transparente para quien la utiliza. Con eso, la red local de la empresa asume una nueva función y es la de proveer flujo de información (Cappiello y Pedrini, 2017).

La función principal de todos los cursos es capacitar al funcionario para que adquiera una nueva competencia, gane calidad en su trabajo y consiga mantener una relación transparente, de lenguaje simple y clara con su cliente, y lo auxilie así con los datos a fin de que pueda tomar la mejor decisión de uso de los productos y servicios ofrecidos por el banco. 


\subsection{Perfil del bancario}

Acerca del perfil del profesional deseado en la era de la gestión del conocimiento, los bancarios investigados consideran muy importantes las competencias orientadas a la dimensión comportamental y estratégica. En esta cuestión era preciso que el funcionario eligiera cuáles, en su opinión, son las cuatro principales competencias esenciales que todo bancario debe poseer.

Las cuatro competencias más elegidas fueron: poseer ética profesional, con el $21 \%$; saber comunicar, con el $18 \%$; tener compromiso, con el $10 \%$; y saber oír, con el $11 \%$. Esto en consideración a que competencias tales como saber actuar, saber movilizar, saber aprender, tener visión estratégica y asumir responsabilidades deben agregar valor económico para la organización y valor social para el individuo. De acuerdo con los planteamientos de Fleury y Fleury (2001), se constata que la percepción de los bancarios participantes de este estudio se encuentra alineada con las competencias del profesional demandadas por las organizaciones contemporáneas.

\subsection{Caracterización de las actividades de educación corporativa}

Sobre las características del aprovechamiento y la frecuencia de participación en los cursos disponibles en la plataforma de la Universidad Corporativa del Banco ALFA, los ítems se presentan en la tabla 2.

Tabla 2. Características de las participaciones en la plataforma de la universidad corporativa

\begin{tabular}{c|c}
\hline Características del aprovechamiento & Frecuencias principales \\
\hline Utilización de recursos tecnológicos & $80 \%$ concuerdan \\
\hline Motivación para participación & $96 \%$ por la política de la empresa \\
\hline Frecuencia de participación & $52 \%$ solo cuando se solicita \\
\hline Número de entrenamientos realizados & $60 \%$ arriba de 15 cursos \\
\hline Tiempo medio de duración de los cursos & $77 \%$ de hasta tres horas \\
\hline
\end{tabular}

Fuente. Elaboración propia.

\subsection{Evaluación de la contribución de las actividades de educación corporativa}

En este ítem se analizó la intensidad de los cambios de orden tecnológico, humano y organizacional en el día a día, la forma y la opinión general sobre cómo se conduce la realización de cursos y las complicaciones en su realización para los encuestados. Sin embargo, se verificó la intención del Banco ALFA frente a la disponibilidad de los contenidos, la contribución al crecimiento personal, profesional y organizacional, sus ventajas y desventajas, las competencias y las habilidades desarrolladas con la realización de los cursos vía intranet y presencialmente.

De acuerdo con el análisis sobre las contribuciones de las actividades basadas en Vergara (2000), la tabla 3 presenta los cambios al considerar las actividades tecnológicas, humanas y organizacionales.

Tabla 3. Cambios observados en el estudio

\begin{tabular}{l|l|l}
\hline \multicolumn{3}{c}{ Cambios según Vergara (2000) } \\
\hline Tecnológicas & Nuevas formas de trabajo & $60 \%$ respondieron como alta \\
\hline Humanas & Compartir responsabilidad y compromiso humano & $60 \%$ respondieron como media \\
\hline Organizacionales & Reducción de los niveles jerárquicos & $40 \%$ respondieron como media \\
\hline
\end{tabular}

Fuente. Elaboración propia con base en Vergara, 2000. 
Se analizaron también la oportunidad de entrenamiento y la capacitación —enfoque a corto plazo-y desarrollo profesional, desarrollo de competencias, evaluaciones, feedbacks e informaciones acerca del desempeño. Los resultados se presentan en la tabla 4.

Tabla 4. Evaluación de la contribución de las actividades de educación corporativa

\begin{tabular}{|c|c|}
\hline $\begin{array}{c}\text { Contribuciones de las actividades de la universidad } \\
\text { corporativa }\end{array}$ & Frecuencias principales \\
\hline $\begin{array}{l}\text { Forma como el banco incentiva la participación en las } \\
\text { capacitaciones }\end{array}$ & $57 \%$ adecuada \\
\hline Dificultades para la realización de las actividades & $88 \%$ aducen falta de tiempo \\
\hline $\begin{array}{l}\text { Percepción sobre las intenciones del banco en cuanto a la } \\
\text { disponibilidad de los cursos }\end{array}$ & $51 \%$ para alcance de metas \\
\hline Contribuciones sobre la realización de los cursos & $64 \%$ creen en la agregación de conocimiento \\
\hline Desventajas observadas sobre la realización de cursos & $\begin{array}{l}75 \% \text { reclaman la falta de tiempo para } \\
\text { desarrollar las actividades } \\
57 \% \text { no observan la aplicabilidad de los } \\
\text { temas abordados }\end{array}$ \\
\hline Ampliación de nuevos conocimientos y temas & $\begin{array}{l}55 \% \text { amplían el conocimiento de manera } \\
\text { positiva, pues se trata de nuevos temas que } \\
\text { contribuyen a la mejoría de la calidad de su } \\
\text { trabajo }\end{array}$ \\
\hline Contribuciones personal y profesional & $\begin{array}{l}69 \text { \% eligieron mejoría de decisión } \\
38 \text { \% creen en la mejoría de la creatividad }\end{array}$ \\
\hline Contribuciones relacionadas a la organización & $50 \%$ en mejoría de los procesos \\
\hline
\end{tabular}

Fuente. Elaboración propia.

De acuerdo con los datos, el $57 \%$ de los encuestados considera adecuadas las actividades de educación corporativa, mientras que el $43 \%$ cree que son parcialmente adecuadas. La parcialidad se justificó con base en dos motivos principales: el corto plazo que los funcionarios reclaman tener para realizarla - seguido de la constante cobranza por parte de los superiores- y el hecho de que las actividades se impusieran durante el horario de trabajo, lo que dificulta su realización inmediata.

Se buscó saber, en lo que concierne a la intensidad del acceso a los cursos, cuál sería la complicación para su realización. En la estructuración del cuestionario se tenía como intención conocer cuál sería la contribución de la educación corporativa según cada funcionario. A la par de esta idea se percibió cuán interesante sería descubrir, para cada funcionario, la intención del banco en tornar disponibles cursos de actualización y capacitación, y si sería esta oportunidad vista con buenos ojos.
Por tanto, a partir de lo que la mayoría cree, la principal intención de la empresa al tornar disponibles los cursos de actualización y capacitación a través de su educación corporativa es alcanzar una ventaja competitiva y hacer que la empresa alcance sus metas, más que proporcionar el desarrollo del funcionario y sus realizaciones personales.

De esta manera, es posible verificar que existe una relación entre el hecho de que el $96 \%$ de los funcionarios afirmaron realizar los cursos apenas en función de la política de la empresa y no por iniciativa propia, y el $51 \%$ cree que los cursos se ofrecen con la intención de direccionamiento estratégico para el alcance de metas. Una vez más, se percibe que los funcionarios encuestados perciben tales oportunidades como algo más benéfico para la empresa que para ellos.

Verificar que los dos mayores porcentajes, esto es, $38 \%$ y $26 \%$, se refieren positivamente a la cuestión, 
refuerza la percepción arriba destacada, pues ambas concuerdan de manera parcial y leve, respectivamente, ya que los cursos ofrecidos por la institución corresponden a su actuación profesional diaria, o sea, condicen con lo que se realiza dentro de las respectivas funciones.

Sobre los cursos se verificó que se administran con el fin de garantizar un perfecto aprovechamiento de lo que se enseña, y una vez más los mayores porcentajes presentan resultados positivos, ya que el $38 \%$ y el $26 \%$ concuerdan de manera leve y parcial, respectivamente, en que los cursos se administran de manera tal que garantizan un perfecto aprovechamiento de lo que se enseña. Esto significa que los esfuerzos de la empresa para que los encuestados consigan obtener un buen aprovechamiento de lo que les enseñan son positivos.

En el análisis esto significa que las competencias requeridas, de acuerdo con los encuestados, están presentes en los cursos o programas ofrecidos, lo que demuestra que la empresa, para la mayoría de los encuestados, acierta en sus intenciones de cursos.

De acuerdo con Seleme y Munhoz (2010), la idea de la educación corporativa exige que su cultura se incorpore en la organización, pues de nada sirve formar colaboradores si las nuevas ideas adquiridas se pierden en el comportamiento tradicional y no se aplican para la mejoría funcional y competitiva de la organización, si no existe cooperación entre los colaboradores y las gerencias estratégicas en la empresa, o si no se realizan actividades de acompañamiento y evaluación de los resultados.

Los cursos de actualización y capacitación tienen como intención desarrollar habilidades y competencias. A partir de esto, se buscó verificar, entre una serie de competencias y habilidades, cuáles, según los encuestados, serían las tres principales que deberían desarrollarse en la realización de los cursos.

La capacidad de liderazgo, con el $28 \%$, fue la principal competencia y la habilidad que a los encuestados les gustaría adquirir con la realización de los cursos, el conocimiento del negocio, con el $25 \%$, ocupó la segunda posición; seguida del desarrollo de la visión estratégica, con el $17 \%$.

Segun Motta (1997), la capacidad que un individuo posee de influenciar a alguien $\mathrm{o}$ a un grupo de personas significa una fuerza psicológica en la que uno actúa de modo que modifica el comportamiento del otro de modo intencional. Esa influencia supone poder y autoridad y altera así el modo de actuar del influenciado.

Grandes liderazgos marcaron el escenario de cada época, pues tuvieron la osadía de implantar de inmediato una idea nueva y asumir, con responsabilidad, las posibilidades de éxito de sus ideas o actitudes. Son personas que poseen normalmente trazos osados y corajosos, flexibles y adaptables, que sepan interferir en su medio para provocar cambios necesarios y deseables de un pueblo o de una determinada nación. Todo líder posee conocimiento de negocio y visión estratégica. Frente a eso, es posible percibir que los funcionarios desean recibir capacitación para el liderazgo y, consecuentemente, a fin de adquirir posiciones de líder buscan los cargos más altos y así ser reconocidos para una promoción.

En un segundo análisis se buscó establecer qué creen adquirir como ventaja y desventaja con la realización de los cursos los encuestados. Las preguntas se contestaron en una escala de 1 a 4 , en la que 1 significa índice de contribución baja, 2 media-baja, 3 regular y 4 alta.

Una vez más la cuestión del tiempo peca en una buena atención a las actividades. Dado que los cursos deben realizarse en el horario de expediente, junto con las funciones cotidianas, la mayoría de los funcionarios cree que los cursos son perjudiciales pues se acumulan con otras tareas que no pueden presentar atraso en su realización.

En la figura 1 se presentan los índices de contribución de las variables sobre el desempeño del entrenamiento de los funcionarios. 
Figura 1. Índice de contribución de cada grupo de variables sobre el desempeño del sitio web de la universidad corporativa

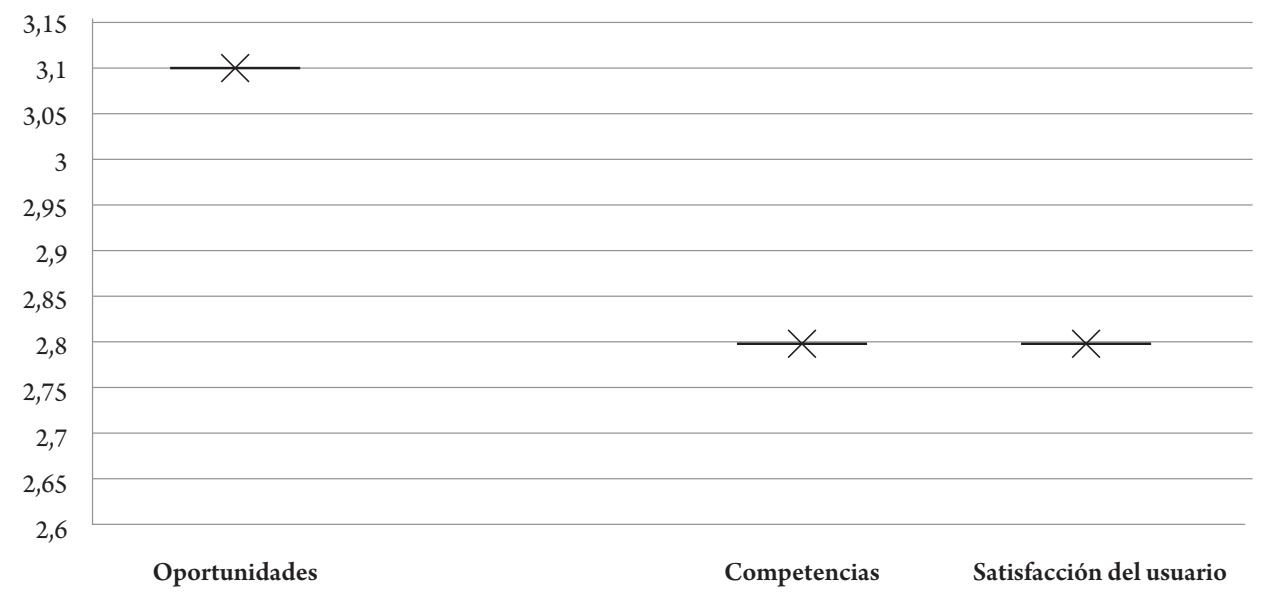

Fuente. Elaboración propia.

Como se observa en la figura 1 , las contribuciones relacionadas con las oportunidades - 3,1- en media son más satisfactorias que las relacionadas con el desarrollo de competencias - 2,8- y la satisfacción del usuario -2,79-. En la figura 1 también se presentan los resultados sobre los encuestados acerca de la existencia de algún levantamiento junto con los funcionarios en relación con las necesidades y las formas de entrenamiento.

Esto demuestra que, de acuerdo con la mayoría de los encuestados, no existe interés de la empresa frente a esa cuestión, lo que puede llegar a ser perjudicial para esta, pues debería utilizar en el perfeccionamiento de los cursos o programas la opinión de los funcionarios que viven el día a día de la empresa y saben identificar en qué aspectos se deben mejorar los procesos.
También se cuestionó si el banco valoriza a los funcionarios que buscan capacitación. Esa respuesta se puede entender frente al hecho de que algunos funcionarios antiguos todavía ocupen cargos en las agencias sin enseñanza superior, con apenas enseñanza media. Parece entonces que algunos funcionarios no requieren cualificación para que los valoren. En la última parte del cuestionario se buscó verificar el grado de satisfacción de los encuestados con relación a varios factores, tales como desarrollo profesional, entrenamiento, auxilios y actividades realizadas.

En la figura 2 se presentan las variables descritas y sus índices de desempeño, resumidas en la figura 1. 
Figura 2. Variables del índice de desempeño con relación al sitio web de la universidad corporativa

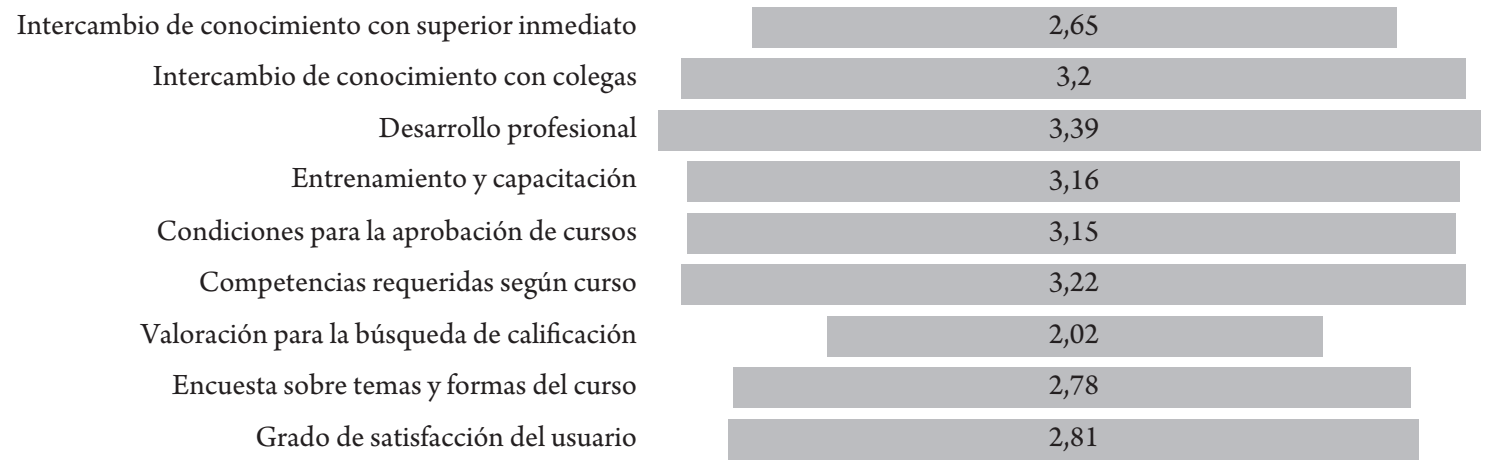

Fuente. Elaboración propia.

\section{Consideraciones finales}

$\mathrm{L}$ a realización de este estudio confirma que las Jorganizaciones, en sus prácticas y procesos relacionados con la gestión del conocimiento y el desempeño de las personas en el espacio productivo, demuestran una tendencia a la migración del entrenamiento y del desarrollo tradicional para la educación corporativa. Con esto ganan enfoque y fuerza estratégica, lo que se evidencia hoy como uno de los pilares de una gestión empresarial exitosa.

Los datos que se recogieron de los encuestados de ocho agencias objeto de este estudio demuestran una comprensión de lo que la organización bancaria adopta como política, y las estrategias de gestión de personas alineadas con el direccionamiento estratégico y que asumen un papel decisivo en la consecución de los resultados pretendidos.

Así, se observa, con respecto al modelo de las competencias, que se utilizaron los recursos pedagógicos con el fin de propagar sus métodos y permitir al trabajador adecuarse de nuevo a un ciclo más de esquemas que se encuadran en una constante búsqueda por la cualificación, el perfeccionamiento y la adecuación a los sistemas productivos. Es importante pensar también que la educación se percibe en este contexto como un medio para alcanzar y propagar estos conceptos del mundo del trabajo, como el modelo de las competencias.
En relación con las actividades de educación corporativa afirman acceder a los cursos vía intranet solo cuando se solicita, única y exclusivamente en función de la política de la empresa.

Los encuestados todavía consideran adecuada la forma en la que el banco procede con la conducción de la participación de las actividades corporativas y afirman recibir informaciones claras sobre las actividades que desempeñan. Así mismo, que los evalúan con criterios claros y recibir feedbacks periódicos sobre su desempeño. Esto muestra el trabajo de una gestión bien realizada en la que existe preocupación sobre el bienestar del funcionario en el interior de aquella empresa. La mayoría aún concuerda en tener oportunidad de entrenamiento, de capacitación y de desarrollo profesional. En cuanto a esto no caben dudas, pues, conforme a lo que se presentó, existe una constante preocupación del banco por poner a disposición entrenamiento y capacitaciones, y es de conocimiento de una gran parte de los funcionarios esa intención.

Para completar, los encuestados todavía no están plenamente satisfechos con los cursos ofrecidos por la institución, pues muchos afirman que el contenido disponible no corresponde a su actuación profesional diaria y no está relacionado con las competencias requeridas para la ejecución de sus tareas. Además, 
no garantizan un perfecto aprovechamiento de lo que se enseña. De esta manera, es preciso que la empresa verifique lo que no realiza de manera correcta, mediante, por ejemplo, feedbacks que se reciban al final de cada curso. Otro factor importante es actualizarse con relación a la opinión de sus funcionarios, ya que la mayoría afirma que no existe ningún levantamiento de información que se realice de la mano de los funcionarios sobre las necesidades y las formas de entrenamiento.

Sin embargo, sin duda la cuestión clave se encuentra en la gran cantidad de reclamos relacionados con la falta de tiempo como el principal problema para la realización de los cursos y las tareas diarias al mismo tiempo, de modo que es necesario que la empresa desarrolle horarios específicos para su realización, y así no se tome esta actividad como algo agotador y obligatorio.

Otro factor importante que es necesario mencionar es que, a pesar del estudio realizado, no se encontró la existencia de una universidad corporativa Banco ALFA, como es el caso de dos importantes organizaciones financieras concurrentes. La falta de este vehículo eficaz en la organización lleva a que la alineación y el desarrollo de los talentos humanos, en relación con las estrategias empresariales y la generación, la asimilación, la difusión y la aplicación del conocimiento organizacional por medio de un proceso de aprendizaje activo y continuo, no sea totalmente eficaz.

\section{Referencias}

Allen, M. (2002). The corporate university handbook: designing, managing, and growing a successful program. Nueva York: Amacom.

Amorim, W. A. C.; Cruz, M. V. G. da; Sarsur, A. M.; Fischer; A. L. (2015). Políticas de educação corporativa e o processo de certificação bancária: distintos atores e perspectivas. $R E A D$ Revista Eletrônica Administrativa, 21(3), 622-647. DOI: https://doi.org/10.1590/1413-2311.0012015.54926

Brandão, G. R. (2006). Gestão de pessoas e as universidades corporativas: dois lados da mesma moeda? RAE, 46(2), 23-33. DOI: https://doi.org/10.1590/S0034-75902006000200003
De esta manera, se puede concluir que no es del todo malo que los funcionarios concuerden parcialmente en que el banco valora los funcionarios que buscan cualificación. Por tanto, poseer capacitaciones presenciales o virtuales para estos encuestados acaba por no ser un diferencial o hacer que el funcionario se desarrolle profesionalmente frente a otro que no los posea. Por tanto, algo como un mayor incentivo a la participación en los programas o cursos financiados por el banco es esencial. Se sugiere que una universidad corporativa evalúe de forma continua el sistema de incentivo.

La mayor dificultad encontrada en la investigación fue la falta de disponibilidad de la colaboración de los bancarios para contestar el cuestionario. Una limitación constatada en el desarrollo de este trabajo se evidenció con relación a su exhaustividad, por tratarse de un tema bastante amplio y subjetivo, en la medida en que depende de la visión, las necesidades y las experiencias de cada individuo con relación a una serie de aspectos, lo que agrega complejidad a su proceso de medición. Todavía con relación a las limitaciones, cabe destacar la objetividad con la que las preguntas abiertas del cuestionario se respondieron, lo que evidencia la dificultad de hacer que los encuestados expongan con mayor profundidad sus opiniones a través de la realización de un cuestionario.
Cappiello, G.; Pedrini, G. (2017). The performance evaluation of corporate universities. Tertiary Education and Management, 23(3), 304-317. DOI: https://doi.org/10.1080/13583883.2 017.1329452

Chaise, O. de F. (2016). Educação corporativa na estratégia organizacional: capacitação de empregados públicos (Tesis de maestría). Universidade de Lisboa, Postugal. Recuperado de https://repositorio.ul.pt/handle/10451/26690

Chiavenato, I. (2009). Comportamiento organizacional-la dinámica del éxito en las organizaciones (trad. P. Mascaró Sacristán). México: McGraw-Hill. 
Dahyana, Y; Susanty, A. I. (2018). Incentive system impact on individual performance through motivation in a corporate university in Indonesia. Sustainable Collaboration in Business, Technology, Information and Innovation (SCBTII), 1(1).

Esteves, L. P.; Meiriño, M. J. (2015). A educação corporativa e a gestão do conhecimento. Ponencia presentada en XI Congresso Nacional de Excelência em Gestão. UFF, Río de Janeiro, Brasil, 13-14 de agosto.

Febraban. (28 de agosto de 2017). Infi-Febraban realiza encontro inédito entre universidades acadêmicas e corporativas. Recuperado de https://bit.ly/2IJ4RlQ

Fleury, A. C. C.; Fleury, M. T. L. (2001). Gestão estratégica do conhecimento. San Pablo: Editora Atlas.

Garbanzo-Vargas, G. M. (2015). Desarrollo organizacional y los procesos de cambio en las instituciones educativas, un reto de la gestión de la educación. Revista Educación, 40(1), 67-87. DOI: https://doi.org/10.15517/revedu.v40i1.22534

Guerci, M.; Bartezzaghi, E.; Solari, L. (2011). Training evaluation in Italian corporate universities: a stakeholder-based analysis. International Journal of Training and Development, 14(1), 291-308. DOI: https://doi.org/10.1111/j.14682419.2010.00359.x

Iglesias, V. S. (2017). Universidad corporativa: una comparativa con la escuela de negocios (Icade Business School-Repsol). Madrid: Facultad de Ciencias Económicas y Empresariales, Icade Business School.

Martino, V.; Scarcella-Prandstraller, S. (2018). Corporate cultural responsibility and shared value in the banking sector. An explorative study in Italy. Communication Management Review, 3(1), 58-79.
Mazariegos, M. I. S. (2015). Motivación y desempeño laboral (Tesis de grado). Facultad de Humanidades, Universidad Rafael Landívar, Guatemala.

Meinster, J. (1999). Educação corporativa: a gestão do capital intelectual através das universidades corporativas. San Pablo: Makron Books.

Montaña, D. M. C.; Labrador, C. R.; Jiménez, C. F. M. (2016). Propuesta diseño modelo universidad corporativa. Bogotá: Universidad Sergio Arboleda, Escuela de Postgrado, Especialización Gerencia Talento Humano.

Moraes, S. A. R. (2016). A educação corporativa como fator estratégico para o comprometimento organizacional: o caso da Universidade dos Correios-Único. Revista Brasileira de Educação e Cultura, (8), 93-114.

Motta, P. R. (1997). A ciência e a arte de ser dirigente ( $8^{\mathrm{a}}$ ed.). Río de Janeiro: Editora Record.

Seleme, R. B.; Munhoz, A. S. (2010). Criando universidades corporativas no ambiente virtual. San Pablo: Pearson Prentice Hall.

Tavares-Filho, J. P.; Bernardes, J. F. (2005). Poder, gobierno y estrategias en las universidades de América del Sur. Ponencia presentada en el $V$ Coloquio Internacional sobre Gestión Universitaria en América del Sur, Mar del Plata, Argentina, 8-10 de diciembre.

Vergara, S. C. (2000). Gestão de pessoas. Sab Pablo: Atlas.

Vega, L. A. O.; Salaiza, F. C. L. (2016). Nuevas formas de organización para la innovación administrativa y técnica. Telos. Revista de Estudios Interdisciplinarios en Ciencias Sociales, 18(1), 121-137. 
